\title{
Multiwall Carbon Nanotube Composites as Artificial Joint Materials
}

Atsushi Sobajima, Takumi Okihara, Shigeaki Moriyama, Naoyuki Nishimura,

Takako Osawa, Kazutaka Miyamae, Hisao Haniu, Kaoru Aoki, Manabu

Tanaka, Yuki Usui, Ken-ichi Sako, Hiroyuki Kato, and Naoto Saito

\section{Supporting Information}




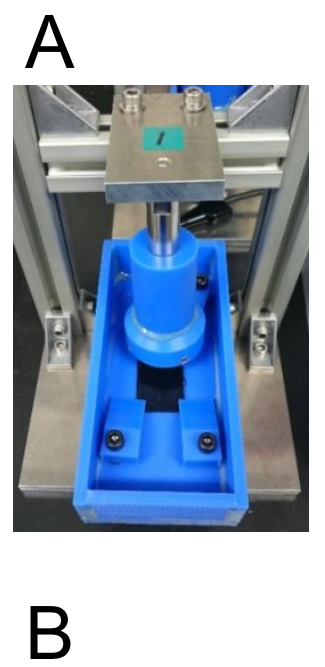

Sliding surface (approximately $1500 \mu \mathrm{m}$ )
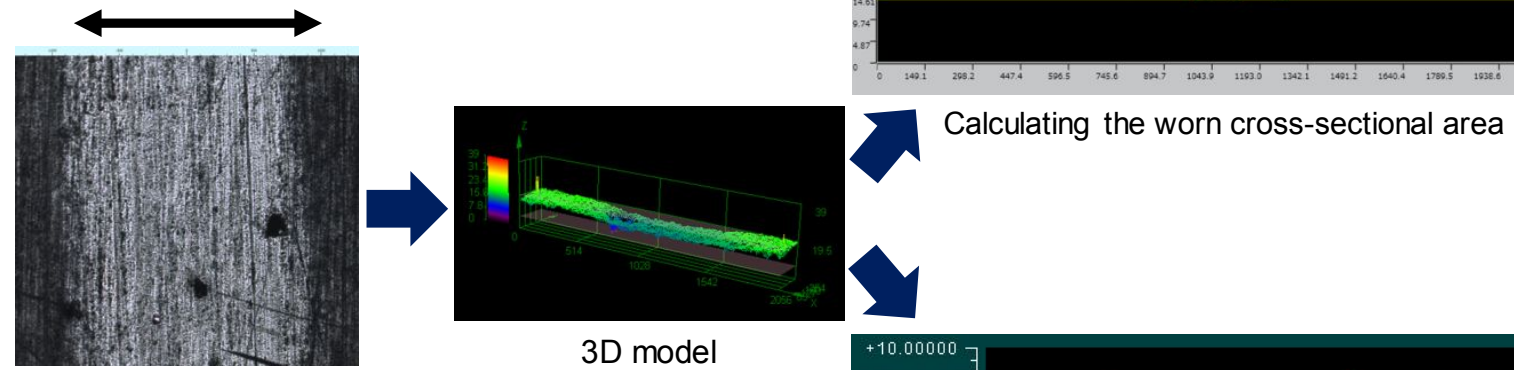

Sample surface 3D model

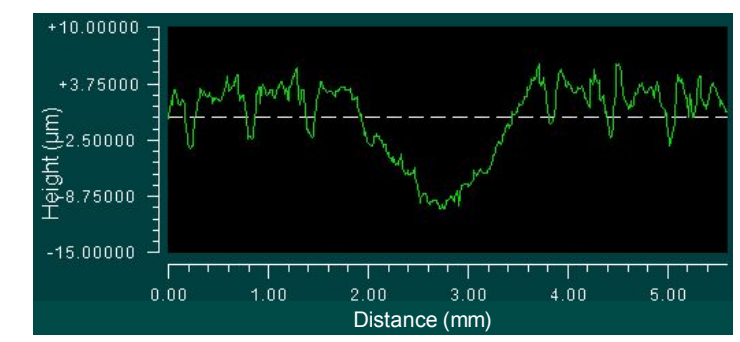

Developing a model of worn cross section

Figure S1. An wear resistance test under ordinary pin-on-disc conditions showed that no quantifiable wear occurred in the MWCNT/UHMWPE composite or the cross-linked UHMWPE. For this reason, we built a sliding tester capable of measuring the area of cross-sectional wear by dry sliding 
under high pressure, and used it to assess the wear resistance. (A) Appearance of the sliding tester. The prepared test piece was inserted into the tester, and a ceramic ball identical to an artificial joint head was subjected to repeated linear reciprocal sliding motions for 24 hours under the following conditions: pressing pressure $15 \mathrm{~N}$, reciprocal distance $40 \mathrm{~mm}$, speed $100 \mathrm{~mm} / \mathrm{sec}$, acceleration $0.2 \mathrm{G}$, deceleration $0.2 \mathrm{G}$, and total traveling distance $3.5 \mathrm{~km}$. (B) Method of assessing wear resistance in a cross-section of MWCNT/UHMWPE composite worn by sliding contact with another surface. To calculate wear resistance, the wear surfaces were profiled, and the wear cross-sectional area corresponding to the amount of wear was measured using a laser microscope (OLS4000-SAT, OLYMPUS, Tokyo, JAPAN) and a 3dimensional optical profiler system (Newview 7300, Zygo Corp., Middlefield, CT, USA). Taking into account plastic deformations and other aspects, the amount of wear was determined by evaluating the worn cross-sectional area, with the mean height at a position sufficiently apart from the sliding surface as the pre-abrasion surface height. Although high-polymer materials may in time regain their original form, our testing detected no such recovery over a 3-month period in similarly measured samples. Thus, we believe that it is 
acceptable to consider the difference in the cross-sectional area as the amount

of wear. 

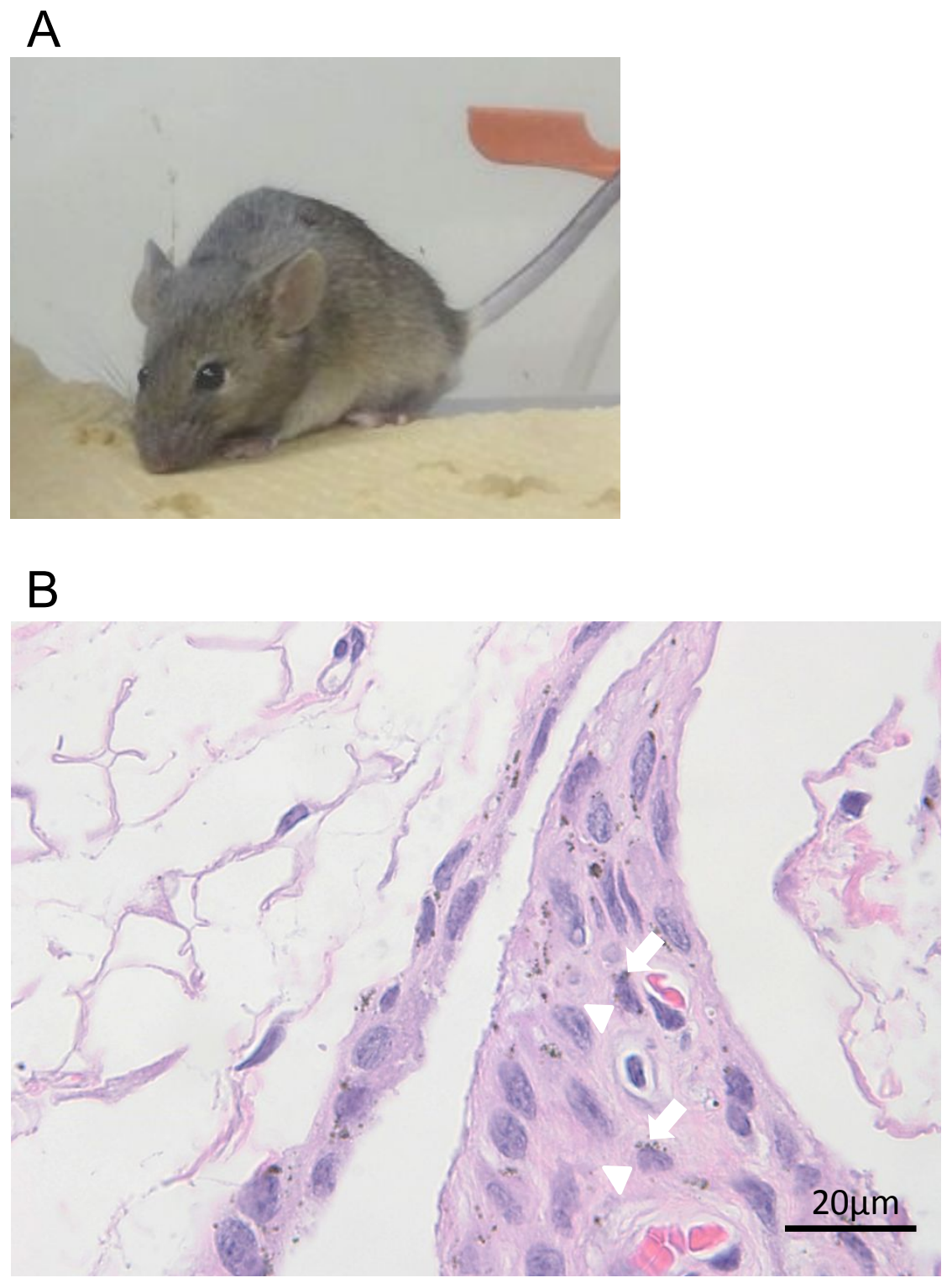

Figure S2. A carcinogenicity study was conducted in rasH2 mice genetically programmed to exhibit significantly increased tumor growth. (A) Appearance of rasH2 mice. (B) At 26 weeks after knee injection of MWCNTS, no tumor developed. MWCNTs were phagocytosed by macrophage-like cells in the joint 
without causing inflammation. Arrows: MWCNTs. Arrowheads: macrophagelike cells. 